\title{
Simple Workflow Changes Enable Effective Patient Identity Matching in Poison Control
}

\author{
Mollie R. Cummins ${ }^{1} \quad$ Pallavi Ranade-Kharkar ${ }^{2} \quad$ Cody Johansen $^{3}$ Heather Bennett ${ }^{4}$ Shelley Gabriel ${ }^{5}$
} Barbara I. Crouch ${ }^{4}$ Guilherme Del Fiol ${ }^{6}$ Matt Hoffman $^{7}$

\footnotetext{
${ }^{1}$ University of Utah College of Nursing, The University of Utah, Salt Lake City, Utah, United States

${ }^{2}$ Homer Warner Center, Intermountain Healthcare, Salt Lake City, Utah, United States

${ }^{3}$ Utah Health Information Network, Murray, Utah, United States

${ }^{4}$ Utah Poison Control Center, The University of Utah, Salt Lake City, Utah, United States

${ }^{5}$ College of Nursing, University of Utah, Salt Lake City, Utah, United States

${ }^{6}$ Department of Biomedical Informatics, The University of Utah,

Salt Lake City, Utah, United States

${ }^{7}$ Medical Informatics, Utah Health Information Network, Murray, Utah, United States
}

Appl Clin Inform 2018;9:553-557.

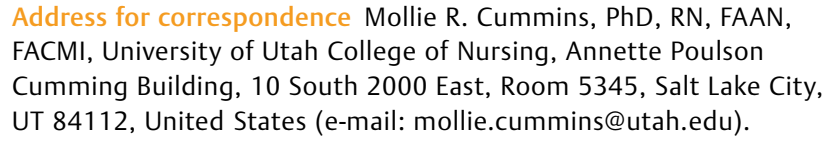

Address for correspondence Mollie R. Cummins, PhD, RN, FAAN, FACMI, University of Utah College of Nursing, Annette Poulson Cumming Building, 10 South 2000 East, Room 5345, Salt Lake City, UT 84112, United States (e-mail: mollie.cummins@utah.edu).

\section{Abstract}

Keywords

- health information exchange

- toxicology

- delivery of health care

- electronic health records
Background U.S. poison control centers pose a special case for patient identity matching because they collect only minimal patient identifying information.

Methods In early 2017, the Utah Poison Control Center (Utah PCC) initiated participation in regional health information exchange by sending Health Level Seven Consolidated Clinical Document Architecture (C-CDA) documents to the Utah Health Information Network and Intermountain Healthcare. To increase the documentation of patient identifiers by the Utah PCC, we (1) adapted documentation practices to enable more complete and consistent documentation, and (2) implemented staff training to improve collection of identifiers.

Results Compared with the same time period in 2016, the Utah PCC showed an increase of $27 \%(p<0.001)$ in collection of birth date for cases referred to a health care facility, while improvements in the collection of other identifiers ranged from 0 to $8 \%$. Automated patient identity matching was successful for $77 \%$ (100 of 130 ) of the C-CDAs.

Conclusion Historical processes and procedures for matching patient identities require adaptation or added functionality to adequately support the PCC use case.

\section{Background and Significance}

Poisoning is the leading cause of injury death in the United States. ${ }^{1}$ Poison control centers (PCCs) play a critical role in the treatment of poisonings by providing consultation to both health care providers and the general public. Of the 2.8 million poison exposures reported to U.S. PCCs in 2015, 29.3\% were managed in a health care facility. ${ }^{2}$ PCCs collaborate daily with emergency departments to provide care for patients. PCCs provide information about the poison, expected clinical effects, monitoring parameters, and advise about specific treatment. Collaboration between PCCs and emergency departments (EDs) currently depends strongly upon telephone communication, allowed under the Health Insurance Portability and Accountability Act of 1996 rule due to the emergent nature of poisoning. We previously identified vulnerabilities and received January 30, 2018 accepted after revision June 2, 2018

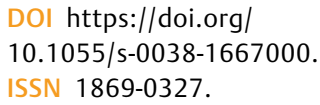

DOI https://doi.org/ 10.1055/s-0038-1667000. ISSN 1869-0327.

(c) 2018 Georg Thieme Verlag KG
Stuttgart . New York

License terms

$($ (1) $\ominus \circledast$ 
inefficiencies in the ED-PCC collaboration system that can lead to miscommunication, data loss, and error, which must be addressed to ensure patient safety. ${ }^{3-5}$ Examples of safety vulnerabilities include: difficulty establishing synchronous verbal communication via telephone, discussion of multiple patients during the same telephone conversation, and communication with nonclinical staff members. Additionally, any information moved among patient care settings via phone may or may not be documented for continued use by the recipient health care facility.

Electronic health information exchange (HIE) is a promising approach to improve communication between EDs and PCCs, addressing vulnerabilities and inefficiencies in the ED-PCC collaboration. To accomplish HIE, it is necessary to match patient records (i.e., identity matching) between systems used at EDs and PCCs. However, given the lack of a universal health identifier in countries such as the United States, patient identity matching is a persistent challenge, especially at settings that are unaccustomed to verifying patient identities, such as U.S. PCCs. Patient identifying information is routinely collected in most health care settings, as it is necessary to support record linkage and HIE in support of care provision, payment, and billing. U.S. PCCs pose a special case for patient identity matching because they collect only minimal patient identifying information, and callers often prefer anonymity.

In early 2017, the Utah PCC initiated participation in regional HIE by sending Health Level Seven Consolidated Clinical Document Architecture (C-CDA) documents to the Utah Health Information Network (UHIN) regional HIE and Intermountain Healthcare (IHC). As part of the implementation, we initiated measures to optimize patient identity matching. In this brief report, we (1) describe the workflow and information management modifications that we implemented to improve the collection of patient identifiers at the Utah PCC, and (2) assess the effect of those changes in the collection of identifiers and patient matching rates. Our approach can be adopted by other PCCs and similar entities that aim to engage in HIE.

\section{Background}

\section{Patient Identity Matching and Health Information Exchange}

Efficient HIE processes depend upon the capability to automatically match patient identities; systems and processes for accomplishing this match are well-developed, if imperfect. Information management processes at hospitals and health care organizations routinely collect pieces of identifying information, such as social security numbers, addresses, and dates of birth, as these types of information are critical for managing a patient's records and accomplish billing and payment. ${ }^{6}$ Registration processes at health care facilities ensure collection of adequate identifiers to accomplish automated identity matching for many patients. The process of exchanging and using these identifiers to match patient records for the purpose of HIE is frequently conducted according to the Nationwide Health Information Network Cross-Community Patient Discovery (XCPD) specification. ${ }^{7}$
This specification requires name, birth date, and gender, and if available and permissible, address, phone number, and social security number. When automated patient identity matching fails, manual effort is required to link records for a unique person.

\section{Poison Control and Patient Identities}

PCCs serve the public and health care providers, providing information and advice in call centers staffed by pharmacists and/or nurses. PCCs do routinely collect identifying information such as name and age. However, since they do not bill for services, they collect minimal identifying information. In sensitive situations such as illegal use of substances or suicide attempts, callers may not wish to disclose any identifying information, or may supply false information, for fear of legal consequences or privacy concerns. This behavior is well documented in the case of heroin overdose, where bystanders are known to fail to call for help, or call then flee the scene, for fear of arrest when police arrive. ${ }^{8}$ In other scenarios, it may not be appropriate to delay emergency care for collection of extensive information over the phone. Therefore, PCCs lack incentives for and face challenges against the implementation of adequate processes for collecting identifying information that can be used for identity matching.

\section{Health Information Exchange at the Utah Poison Control Center}

Our team at The University of Utah developed a process, workflow, and purpose-built software (SNOWHITE) that enables PCC participation in HIE. ${ }^{9-11}$ We implemented the process and software at the Utah PCC in March 2017, and we are actively creating and sending C-CDA consultation notes to the UHIN for all cases referred to a health care facility. UHIN, in turn, links the C-CDAs to individual patient records in the Utah statewide clinical HIE (CHIE). From the CHIE, the notes can be routed to any participating health care organization, or accessed via login by an authorized user. Additionally, UHIN provides discharge summaries for patients with a Utah PCC C-CDA. We have created the necessary informatics tools, HIE processes, and workflow processes necessary to accomplish HIE with PCCs. However, the capability of this process to positively impact patient care is largely dependent upon our ability to match up the communication with a specific patient, through a process of patient identity matching.

\section{Patient Identity Matching by Regional HIE}

UHIN uses a master patient index (MPI) to capture and store identifying information about individuals who receive care at UHIN member facilities. UHIN's MPI encompasses 408 facilities in the state of Utah, and includes 5.9 million patients. Examples of demographic identifiers stored in the MPI include: basic demographic information (e.g., name, address, phone number), date of birth (DOB), patient identifying numbers (e.g., medical record number, insurance member ID), gender, and social security number. For identity matching, UHIN uses proprietary software (IBM Initiate) that implements the Fellegi-Sunter probabilistic method. Approaches based on the Fellegi-Sunter probabilistic method use the frequencies of values of each field 
within the population as a basis of a computed score. ${ }^{12}$ The higher the score of a match, the higher the likelihood that the match is correct. A similar approach is adopted by most HIEs nationwide.

\section{Methods}

\section{Preliminary Assessment}

In preparation for HIE, we performed a preliminary assessment of the rate of successful patient identity matching between the Utah PCC and the electronic health record (EHR) system at IHC. We retrospectively analyzed successful PCC referrals to IHC EDs for the year 2015. In a successful PCC referral to an ED, the PCC refers a patient to an ED for further evaluation and treatment, and the patient complies with the recommendation. We performed XCPD transactions between the Utah PCC and the IHC EHR using the patient identity information stored in ToxiCALL, the data collection system used at the Utah PCC and several other PCCs in the United States. For those referrals in 2015 , only $7 \%$ of the patients successfully matched between the two systems. Manual review of the data set indicated that missing DOB, patient phone number, and incomplete address were the primary reasons for the low match rate. We found that the patient's name and gender, and caller phone number were the only pieces of identifying information routinely recorded in the system by PCC specialists. DOB, a critical data element for accurate identity matching, was not routinely captured. Patient address and patient phone number were rarely collected. In fact, the PCC information system has no structured field dedicated to recording a patient's DOB.

Adapting PCC Workflow and Documentation Practices To increase the documentation of information necessary for patient identity matching, we (1) adapted documentation practices to enable more complete and consistent documentation of patient identifiers, and (2) implemented staff training to improve collection of identifiers. Training efforts included large group training sessions, superuser training, and one-on-one coaching of staff. We also created a user guide and displayed reminders to document DOB on wallmounted screens located at the PCC.

The collection of DOB, an important identifier, required substantial attention. Unable to modify the proprietary Utah PCC information system, we designated a general field for documentation of DOB, and this general field lacks constraints on format. To mitigate the possibility of error in date format, we trained staff to enter data using a consistent date format, through training sessions and posted reminders. We measured the patient matching rate and frequency of identifiers collected during the same 6-week period before (July 13-August 25, 2016) and after (July 13-August 25, 2017) changes were implemented. A wash-out period was introduced to allow stabilization of the process, since during the initial months, the collection and documentation of identifiers may have been inconsistent, as we transitioned from a group of pilot users responsible for sending all C-CDAs to center-wide use.
Table 1 Frequency and percentage of collected patient identifiers for cases referred to a health care facility, before and after workflow modification/training

\begin{tabular}{|l|l|l|l|l|l|l|}
\hline \multirow{2}{*}{ Identifier } & \multicolumn{2}{|l|}{$\begin{array}{l}\text { 2016 } \\
(N=282)\end{array}$} & \multicolumn{2}{l|}{$\begin{array}{l}\text { 2017 } \\
(N=299)\end{array}$} & \multirow{2}{*}{$X^{2}(1)$} & $p$-Value \\
\cline { 2 - 6 } & $n$ & $\%$ & $n$ & $\%$ & & \\
\hline First name & 256 & 91 & 289 & 97 & 7.64 & 0.003 \\
\hline Last name & 246 & 87 & 279 & 93 & 5.48 & 0.003 \\
\hline $\begin{array}{l}\text { Date of } \\
\text { birth }\end{array}$ & 153 & 54 & 243 & 81 & 47.57 & 0.000 \\
\hline $\begin{array}{l}\text { Patient } \\
\text { phone } \\
\text { number }\end{array}$ & 111 & 39 & 136 & 45 & 1.983 & 0.080 \\
\hline Zip code & 82 & 29 & 111 & 37 & 3.88 & 0.024 \\
\hline $\begin{array}{l}\text { Street } \\
\text { address }\end{array}$ & 0 & 0 & 0 & 0 & N/A & N/A \\
\hline City & 84 & 30 & 111 & 37 & 3.18 & 0.037 \\
\hline State & 84 & 30 & 114 & 38 & 4.13 & 0.021 \\
\hline
\end{tabular}

a "2016" indicates the time period July 13-August 25, 2016, and "2017" indicates the time period July 13-August 25, 2017.

\section{Results}

During the 6-week time period, 81\% (243/299) of Utah PCC records corresponding to patients referred to a health care facility contained a documented, correctly formatted DOB. Compared with the same time period in 2016, Utah PCC showed a $27 \%$ increase $(p<0.001)$ in the collection of DOB for cases referred to a health care facility, while improvements in the collection of other identifiers ranged from 0 to $8 \%$ (see - Table 1). After the implementation of improved patient identity documentation processes, successful automated identity matching increased from 7 to $77 \%$ (100 of 130) of the C-CDAs submitted during the same 6-week time periods in 2016 and 2017, respectively. The remainder of the C-CDAs resulted in either no match or multiple possible matches. UHIN forwarded all C-CDAs to the destination health care organization, as specified by Utah PCC personnel within the C-CDA. However, storage in the cHIE was limited to C-CDAs that matched to the UHIN MPI.

\section{Discussion}

With modification to workflow and documentation practices, we achieved modestly improved rates of identifier documentation. However, the modestly improved rates of identifier documentation and use of a statewide MPI were sufficient to accomplish a patient identity matching rate of $77 \%$, a rate much higher than the $7 \%$ rate found in our preliminary analysis conducted using the MPI at IHC. There are several reasons why the patient identity matching rate was not higher than $77 \%$. As previously mentioned, it is not always feasible for the PCCs to collect a complete set of identifiers, given the emergency circumstances of many poison exposures, and in some situations, callers supply false identifying information. Data entry errors are possible. If a patient is newly seeking care at a 
Utah health care facility, and has not yet completed registration, a match would not be possible. And lastly, demographic information in the statewide MPI could be incorrect.

Our approach required only minimal modification to existing information systems and no programming changes. Therefore, the proposed workflow and information management modifications are feasible for implementation by any PCC that plans to engage in HIE with EDs. Our approach could also be adopted by any setting that does not bill third-party payers (e.g., cosmetic surgery center, free health clinic) and therefore may not collect adequate identifiers to ensure appropriate routing of patient information.

We anticipate that point-to-point patient identity matching (between the Utah PCC and a specific health care facility) would be more successful. During a telephone call, PCCs document the patient's destination ED. Even lacking a robust set of identifiers, a patient presenting to the destination ED an hour later, with partial match of demographic identifiers and matched exposure characteristics, is a likely match. In the future, it is important that automated patient matching algorithms evolve to incorporate such relevant clinical context. In the interim, manual processes implemented during patient registration or triage may be a simple and reliable means to accomplish a definitive match, especially given the manageable proportion of ED cases that are classified as poison exposures. Currently, we are including specific health care facilities as intended recipients on outgoing Utah PCC C-CDAs, so that these documents can be routed to specific destination health care facilities in the absence of a definitive automated patient match. This process mimics the usual telephone-based process for information sharing, in which the PCC specialist calls ahead the ED to which the patient indicated he/she is proceeding.

\section{Limitations}

First, despite improvements, a substantial proportion (nearly 1 in 5) of C-CDAs originating from the Utah PCC required further manual review for accurate resolution at the regional HIE. Matches were not manually validated, and the rate of duplicate records and false-positive records associated with poison control C-CDAs at destination health care facilities remains to be seen. Additional efforts are needed to improve the collection of the patient's phone number and complete address with postal code, as these identifiers remained sparsely populated. This may well be due to the time required to collect these additional identifiers over the phone in the midst of a medical emergency. Second, due to the black box nature of the proprietary identity matching algorithm used at UHIN, we were unable to determine the contribution of each type of identifier to the overall increase in identity matching rate. Third, the 6 -week evaluation period is relatively short, thus we were unable to determine if improvements in the collection of identifiers will sustain over time.

\section{Conclusion}

Incomplete or inaccurate patient identifying information is consistently problematic in patient identity matching, and poses a special case and challenge for HIE processes that involve PCCs. The current systems for patient identity matching may require augmentation for the PCC use case, because patient matching in an emergency care scenario must occur quickly and accurately to avoid medical error. Historical processes and procedures for matching patient identities require adaptation or added functionality to adequately support the PCC use case. In this project, relatively simple modifications to workflow and documentation, and use of a statewide MPI, enabled successful patient identity matching.

\section{Clinical Relevance Statement}

U.S. poison control centers, new participants in standardsbased health information exchange, pose a challenge for existing processes of patient information management and health information exchange. This article describes challenges and outcomes of accomplishing patient identity matching for poison control center patients. As health information exchange grows to encompass a wider variety of participants and exchange scenarios, we are challenged to adapt our systems, tools, and processes.

\section{Multiple Choice Questions}

1. Health care organizations collect patient identifiers to manage a patient's record and accomplish billing and payment. In the process of HIE, patient matching is frequently conducted according to what profile?
a. Health Level Seven (HL7) standards
b. Patient Identifier Cross Referencing Integration (PIX)
c. Cross-Community Patient Discovery (XCPD)
d. Patient Administration Management (PAM)

Correct Answer: The correct answer is option c. Cross Community Patient Discovery (XCPD) is an HIE profile that supports (1) locating communities that hold patient relevant health data, and (2) translating those patient identifiers across communities holding the same patient's data. $\mathrm{XCPD}$ is often used across a regional area, sending many queries to many different systems (such as hospitals and statewide HIE organizations), as opposed to sending one query to a centralized system and receiving back a list of matching results. In the former case, the demographic information is collected and consolidated into one answer.

2. Since patient identifiers are often collected for the primary purpose of billing, what types of health care settings may not collect adequate patient identifiers?

a. Settings that bill insurance companies.

b. Settings that serve Medicare/Medicaid patients.

c. Settings that do not bill third-party payors for services.

d. Settings affiliated with large health care organizations.

Correct Answer: The correct answer is option c. Settings that do not bill third-party payors for services. Unless a health care setting has already adapted its intake processes and documentation to collect identifiers for the 
purpose of billing, it may not collect sufficient patient identifiers for health information exchange.

\section{Protection of Human and Animals Subjects}

This study was reviewed and approved by the University of Utah Institutional Review Board.

\section{Funding}

This study was supported by the U.S. Department of Health and Human Services, Agency for Healthcare Research and Quality, grant 5R01HS021472, and the Office of the National Coordinator for Health Information Technology $(901 \times 003)$. The authors wish to acknowledge the assistance of the Utah Poison Control Center, Intermountain Healthcare, the Utah Health Information Network, and Dr. Tom H. Greene. Additional assistance was provided by the Center for Clinical and Translational Sciences of the National Institutes of Health under Award Number UL1TR001067. The content is solely the responsibility of the authors and does not necessarily represent the official views of the National Institutes of Health (NIH). Support and resources were also provided from the Center for High Performance Computing at The University of Utah, partially funded by the NIH Shared Instrumentation Grant 1S100D021644-01A1.

\section{Conflict of Interest}

None.

\section{References}

1 National Center for Health Statistics. NCHS Data on Drug-poisoning Deaths. NCHS Fact Sheet. August, 2017. Available at: https:// www.cdc.gov/nchs/data/factsheets/factsheet_drug_poisoning. pdf. Accessed July 12, 2018
2 Mowry JB, Spyker DA, Brooks DE, Zimmerman A, Schauben JL. 2015 annual report of the American Association of Poison Control Centers' National Poison Data System (NPDS): 33rd annual report. Clin Toxicol (Phila) 2016;54(10):924-1109

3 Cummins MR, Crouch B, Gesteland P, et al. Inefficiencies and vulnerabilities of telephone-based communication between U. S. poison control centers and emergency departments. Clin Toxicol (Phila) 2013;51(05):435-443

4 Caravati EM, Latimer S, Reblin M, et al. High call volume at poison control centers: identification and implications for communication. Clin Toxicol (Phila) 2012;50(08):781-787

5 Cummins MR, Crouch BI, Del Fiol G, Mateos B, Muthukutty A, Wyckoff $A$. Information requirements for health information exchange supported communication between emergency departments and poison control centers. AMIA Annu Symp Proc 2014; 2014:449-456

6 Morris GFG, Afzal S, Robinson C, Greene J, Coughlin C. Patient identification and matching final report. Final Report ed: Office of the National Coordinator for Health Information Technology; 2014:1-93

7 Godlove T, Ball AW. Patient matching within a health information exchange. Perspect Health Inf Manag 2015;12:1g

8 Davidson PJ, Ochoa KC, Hahn JA, Evans JL, Moss AR. Witnessing heroin-related overdoses: the experiences of young injectors in San Francisco. Addiction 2002;97(12):1511-1516

9 Del Fiol G, Crouch BI, Cummins MR. Data standards to support health information exchange between poison control centers and emergency departments. J Am Med Inform Assoc 2015;22(03): 519-528

10 Nelson SD, Del Fiol G, Hanseler H, Crouch BI, Cummins MR. Software prototyping: a case report of refining user requirements for a health information exchange dashboard. Appl Clin Inform 2016;7(01):22-32

11 Khalifa A, Del Fiol G, Cummins MR. Public health data for individual patient care: mapping poison control center data to the C-CDA consultation note. AMIA Annu Symp Proc 2017; 2016:1850-1859

12 Fellegi IP, Sunter AB. A theory for record linkage. J Am Stat Assoc 1969;64(328):1183-1210 